\title{
Instantons As Unitary Spin Maker
}

\author{
M. Napsuciale ${ }^{\mathrm{a}}$, A. Wirzba ${ }^{\mathrm{b}, *}$, M. Kirchbach ${ }^{\mathrm{c}}$ \\ ${ }^{a}$ Instituto de Fisica, Universidad de Guanajuato, \\ AP E-143, 37150, Leon, Guanajuato, Mexico \\ ${ }^{\mathrm{b}}$ Institut für Kernphysik (Theorie), FZ Jülich, \\ D-52425 Jülich, Germany \\ ${ }^{\mathrm{c}}$ Escuela de Fisica, Univ. Aut. de Zacatecas, \\ AP C-580, Zacatecas, ZAC 98068 Mexico
}

\begin{abstract}
We investigate the relevance of the instanton-induced determinantal 't Hooft interaction to the $\eta$-nucleon coupling $g_{\eta N N}$ within the framework of a three-flavor linear sigma model in the OZI-rule-respecting basis $\left\{(\bar{s} s), \frac{1}{\sqrt{2}}(\bar{u} u+\bar{d} d)\right\}$. Instantons, in combination with the spontaneous breaking of chiral symmetry, are shown to provide the major mechanism for the ideal mixing between pseudoscalar strange and non-strange quarkonia. As long as 't Hooft's interaction captures most of the basic features of the axial QCD gluon anomaly, we identify the anomaly as the main culprit for the appearance of octet flavor symmetry in the anomalous sectors of the pseudoscalar (and axial vector) mesons. Within this context, unitary spin is shown to be an accidental symmetry due to anomalous gluon dynamics rather than a fundamental symmetry in its own right. Though we find the $\eta$-nucleon coupling constant $g_{\eta N N}$ to obey a Goldberger-Treiman like relation, the latter does not take its origin from a pole dominance of the induced pseudoscalar form factor of the octet axial current, but from a subtle flavor-mixing mechanism that is traced back to instanton dynamics. The model presented allows for possible generalizations to non-ideal mixing angles and different values of the meson decay constants in the strange and non-strange sectors, respectively. Finally, we discuss the issue as to what extent the $\eta$ meson may be considered as a Goldstone boson under the constraints of the anomaly-produced unitary spin.
\end{abstract}

Key words: flavor symmetry, eta meson, instantons, OZI rule, $\eta N$ coupling PACS: $11.30 . \mathrm{Hv}, 11.40 . \mathrm{Ha}, 14.40 . \mathrm{Aq}$

\footnotetext{
* Corresponding author.

Email addresses: mauro@feynman.ugto.mx (M. Napsuciale), a.wirzba@fz-juelich.de (A. Wirzba), kirchbach@chiral.reduaz.mx (M. Kirchbach).
} 


\section{Introduction}

Flavor symmetry is presently understood on the basis of QCD and the structure of the quark mass matrix. In the zero quark mass limit, the light flavor sector of QCD acquires a three flavor chiral symmetry $U(3)_{L} \times U(3)_{R}$ which at the level of hadrons is assumed to be realized in the Goldstone phase. The associated Goldstone bosons are identified with the lightest pseudoscalar mesons.

The first problem one encounters in that scheme is the large mass of the $\eta^{\prime}$. The way out of this problem is to take into account quantum corrections which spoil the conservation of the singlet axial current. Particularly relevant to this problem is the existence of Euclidean solutions with non-trivial topological properties (instantons) which also break the $\mathrm{U}(1)_{A}$ symmetry. In fact, the most appealing explanation of the problem of the large $\eta^{\prime}$ mass is provided by the instanton induced quark-quark interaction [1].

The second problem concerns the different symmetries for pseudoscalar and axial vector mesons, on the one side, and vector and tensor mesons, on the other side.

Isoscalar vector mesons closely follow the flavor basis structure: $\phi=\bar{\psi} \lambda_{\mathrm{s}} \psi=\bar{s} s$ and $\omega=\bar{\psi} \lambda_{\mathrm{ns}} \psi=(\bar{u} u+\bar{d} d) / \sqrt{2}$. This contrasts the singlet-octet pattern followed by the isoscalar axial vector and pseudoscalar mesons. To explain this dilemma, the Okubo-Zweig-Iizuka (OZI) rule [2-4] was invoked that forbids the mixing of of quarkonia made of $u$ or $d$ quarks with quarkonia made of $s$ or $c$ quarks. In the absence of symmetry breaking terms one can freely use any basis for the generators of the group. The physically interesting basis is the one whose generators still reflect a residual symmetry of the system in the presence of symmetry breaking terms. In that regard, we have three sources of breaking $U(3)_{L} \times U(3)_{R}$ symmetry. The first one is the $U_{A}(1)$ symmetry breaking by quantum effects. Although the instanton induced interaction is suppressed at high energies due to the factor $\exp \left(-8 \pi^{2} / g^{2}\right)$, it is always present and even becomes decisive at low energies. The second source is the spontaneous breaking of chiral symmetry occurring at the scale $\lambda_{\chi} \approx 4 \pi f_{\pi}$. The third source is the non-vanishing quark mass matrix that explicitly violates three flavor chiral symmetry.

Isospin is a good symmetry for hadronic strong interactions, and in the isospin limit the quark mass matrix simplifies to $M_{q}=\operatorname{Diag}\left(m, m, m_{s}\right)$. This matrix can be written in terms of the $U(3)$ (not of $S U(3)$ ) generators. Using $S U(3)$ would require to consider simultaneously two irreducible representations, the singlet and the octet.

The problem of the conflicting flavor symmetries of the anomalous- and anomaly- 
free meson sectors was addressed e.g. in Ref. [5]. There, the (three) flavor symmetry for hadrons was concluded from the conserved vector current rather than from mass relations and shown to be $S U(2)_{u d} \otimes S U(2)_{c s} \otimes U\left(1_{u d c s}\right)$ in the limit of heavy spectator $c$ quarks. Apparently, this symmetry respects, from the very beginning, the quark generations and the OZI rule. Within the framework of $S U(2)_{u d} \otimes S U(2)_{c s} \otimes U\left(1_{u d c s}\right)$, the $S U(3)$ flavor symmetry for the pseudoscalar and axial vector mesons appears as an artifact of the axial $U(1)_{A}$ gluon anomaly.

It is the goal of the present study to show that unitary spin is an accidental symmetry (in the language of Ref. [6]) that is manufactured by the instanton dynamics, and to explore the implications for the $\eta N$ coupling.

In the next section we shall illustrate the formation of the "eightfold way" within a linear sigma model which has 't Hooft' s determinantal flavor-dependent interaction built in. We will analyze the resulting consequences for the $\eta N N$ coupling constant in Section 3. The paper ends with a brief summary.

\section{2 "Eightfold Way" from instanton dynamics.}

Let us first state the notation for the wave function of the $\eta$ :

$$
\begin{aligned}
|\eta\rangle & =\cos \theta_{P}\left|\eta^{8}\right\rangle-\sin \theta_{P}\left|\eta^{1}\right\rangle, \\
\left|\eta^{8}\right\rangle & =\frac{1}{\sqrt{6}}(\bar{u} u+\bar{d} d-2 \bar{s} s), \quad\left|\eta^{1}\right\rangle=\frac{1}{\sqrt{3}}(\bar{u} u+\bar{d} d+\bar{s} s)
\end{aligned}
$$

Here, $\left|\eta^{8}\right\rangle$ and $\left|\eta^{1}\right\rangle$ are in turn the Gell-Mann's octet and singlet $0^{-+}$states, respectively. The mixing angle $\theta_{P}$ is obtained on the grounds of quadratic $\left(\theta_{P} \approx-10.1^{\circ}\right)$, or linear $\left(\theta_{P} \approx-20^{\circ}\right)$ meson-mass formulae [7]. Obviously, the non-strange quarkonium state $\left|\eta^{\text {ns }}\right\rangle$ is recovered in Eq. (1), when the mixing angle takes the "ideal" value of $\theta_{P}=\theta_{\text {id }}[8]$ with $\theta_{\text {id }} \approx-54.7^{\circ}\left(\cos \theta_{\text {id }}=\right.$ $\left.1 / \sqrt{3}, \sin \theta_{\mathrm{id}}=-\sqrt{2 / 3}\right)$.

Alternatively, in the OZI (generation) basis, the $\eta$ wave function takes the form $\square$

$$
\begin{aligned}
& |\eta\rangle=\cos \phi_{P}\left|\eta^{\mathrm{ns}}\right\rangle-\sin \phi_{P}\left|\eta^{\mathrm{s}}\right\rangle \\
& \left|\eta^{\mathrm{ns}}\right\rangle=\frac{1}{\sqrt{2}}(\bar{u} u+\bar{d} d), \quad\left|\eta^{\mathrm{s}}\right\rangle=\bar{s} s .
\end{aligned}
$$

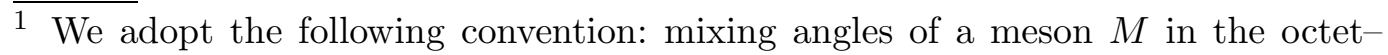
singlet basis are denoted as $\theta_{M}$ and in the non-strange-strange basis as $\phi_{M}$. 
A value of $\phi_{P} \approx 39.4^{\circ}$ has recently been concluded from fitting $\eta\left(\eta^{\prime}\right)$ decay cross sections $[9,10]$. The $\eta^{8}$ state can be recovered from Eq. (3) if $\phi_{P}=\phi_{\mathrm{su}(3)} \approx$ $54.7^{\circ}\left(\sin \phi_{\mathrm{su}(3)}=\sqrt{2 / 3}, \quad \cos \phi_{\mathrm{su}(3)}=\sqrt{1 / 3}\right)$ is inserted instead. The angles in the two bases are related as $\theta_{P}=\phi_{P}-54.7^{\circ}$ in such a way that the physical mixing angle $\phi_{P} \approx 39.4^{\circ}$ in the OZI basis corresponds to the value $\theta_{P} \approx-15.3^{\circ}$ in the octet-singlet basis and thereby lies between the quadratic and linear mixing angles.

To study the formation of the "eightfold way" in an explicit framework, we introduce here a chirally symmetric $\left[U(3)_{L} \otimes U(3)_{R}\right]$ lagrangian for a scalar and a pseudoscalar mesonic nonet, in turn denoted by $\left(\sigma_{i}\right)$ and $\left(P_{i}\right)$,

$$
\mathcal{L}=\mathcal{L}_{\text {sym }}+\mathcal{L}_{U_{A}(1)}+\mathcal{L}_{S B},
$$

where the lagrangian $\mathcal{L}_{\text {sym }}$ describes the flavor-symmetric part, the lagrangian $\mathcal{L}_{U_{A}(1)}$ the flavor-breaking from the $U_{A}(1)$ gluon anomaly, and the lagrangian $\mathcal{L}_{S B}$ the explicit symmetry breaking.

The flavor-symmetric lagrangian $\mathcal{L}_{\text {sym }}$ is given by

$$
\begin{aligned}
\mathcal{L}_{\text {sym }}= & \frac{1}{2} \operatorname{tr}\left[\left(\partial_{\mu} M\right)\left(\partial^{\mu} M^{\dagger}\right)\right]-\frac{\mu^{2}}{2} X(\sigma, P) \\
& -\frac{\lambda}{4} Y(\sigma, P)-\frac{\lambda^{\prime}}{4} X^{2}(\sigma, P),
\end{aligned}
$$

where $M \equiv \sigma+i P$, and $X, Y$ stand in turn for the left-right symmetric traces

$$
\begin{aligned}
& X(\sigma, P) \equiv \operatorname{tr}\left[M M^{\dagger}\right]=\operatorname{tr}\left[\sigma^{2}+P^{2}\right] \\
& Y(\sigma, P) \equiv \operatorname{tr}\left[\left(M M^{\dagger}\right)^{2}\right]=\operatorname{tr}\left[\left(\sigma^{2}+P^{2}\right)^{2}+2\left(\sigma^{2} P^{2}-(\sigma P)^{2}\right)\right] .
\end{aligned}
$$

The pseudoscalar and scalar matrix fields $P$ and $\sigma$ are written in terms of a specific basis spanned by seven of the standard Gell-Mann matrices, namely $\lambda_{i}(i=1, \ldots, 7)$, and by two unconventional matrices $\lambda_{\mathrm{ns}}=\operatorname{diag}(1,1,0)$, and $\lambda_{\mathrm{s}}$ $=\sqrt{2} \operatorname{diag}(0,0,1)$, respectively. The decomposition obtained in this way reads $P \equiv \frac{1}{\sqrt{2}} \lambda_{i} P_{i}$ with $i=n s, s, 1, \ldots, 7$ and similarly for the scalar field.

As we will show below, the main effect in furnishing the masses of the isoscalarpseudoscalar mesons comes from the axial $U_{A}(1)$ gluon anomaly. There exist two candidates for the phenomenological description of the above effect that are associated with two different contributions to $\mathcal{L}$ that lead to $U(1)_{A}$ symmetry breaking at the level of QCD. The first one is the flavor determinantal interaction

$$
\mathcal{L}_{U_{A}(1)}=\mathcal{L}_{I N S T}=-\beta Z(\sigma, P),
$$


where

$$
\begin{aligned}
Z(\sigma, P)= & \left\{\operatorname{det}(M)+\operatorname{det}\left(M^{\dagger}\right)\right\} \\
= & \frac{1}{3}\left\{\operatorname{tr}[\sigma]\left((\operatorname{tr}[\sigma])^{2}-3(\operatorname{tr}[P])^{2}\right)-3 \operatorname{tr}[\sigma] \operatorname{tr}\left[\sigma^{2}-P^{2}\right]\right. \\
& \left.\quad+6 \operatorname{tr}[P] \operatorname{tr}[\sigma P]+2 \operatorname{tr}\left[\sigma\left(\sigma^{2}-3 P^{2}\right)\right]\right\} .
\end{aligned}
$$

It stands for the bosonized 't Hooft effective quark-quark interaction which is induced by instantons (gluon configurations with integer topological charge) and turns out to be determinantal and flavor dependent $[1,11]$. The other candidate is the Veneziano-Witten interaction term

$$
\mathcal{L}_{U_{A}(1)}=\mathcal{L}_{V W}=\beta^{\prime} V W(\sigma, P)
$$

where

$$
V W(\sigma, P)=\left(\ln \operatorname{det}(M)-\ln \operatorname{det}\left(M^{\dagger}\right)\right)^{2},
$$

which takes its origin from the fluctuations of the topological charge (the "ghost pole" mechanism) [12-15]. Consequences of this mechanism were investigated at the level of the fundamental theory in [16] where axial Ward identities were exploited to extract information on pseudoscalar mesons. However, there exists a relation between these two mechanisms for the $U_{A}(1)$-breaking. Indeed, by assuming a non-vanishing vacuum expectation value of $M$ and expanding in powers of the "fluctuation" $\Delta(\operatorname{det} M)=\operatorname{det} M-\langle\operatorname{det} M\rangle$ the authors of Ref. [14] showed that both effective interactions can be linked as

$$
V W-(\operatorname{det}\langle M\rangle)^{-2} Z^{2}=U(3)_{L} \otimes U(3)_{R} \text { invariant operator. }
$$

The first term on the l.h.s. of Eq. (12) can even be related to $Z$ itself by a further expansion in powers of the fluctuation [14]. Clearly, these are not the only possibilities for the breaking $U_{A}(1)$ symmetry. Other possibilities arising from different approximations in the calculational schemes of instanton effects have been proposed [17]. In the present work we restrict ourselves to the usage of the effective 't Hooft interaction. At the phenomenological level it is a promising candidate not only for providing a mechanism of the flavor-mixing but also for simultaneously explaining the unusual properties of scalar mesons, including the long standing problem of the two-photon decays of the $a_{0}(980)$ and $f_{0}(980)[18,19]$.

Finally, the model lagrangian (5) contains the standard term

$$
\mathcal{L}_{S B}=\operatorname{tr}[c \sigma]
$$


which breaks the left-right symmetry explicitly. The $c$ matrix is spanned by the same basis $c \equiv \frac{1}{\sqrt{2}} \lambda_{i} c_{i}$ as the fields, and the nine expansion coefficients $c_{i}$ are independent constants. The most general $c$-matrix that preserves isospin, respects PCAC and is consistent with the quark mass matrix, has $c_{\mathrm{s}}$ and $c_{\mathrm{ns}}$ as the only non-vanishing entries. While the $c_{\mathrm{s}}$ term explicitly causes $\left[U(3)_{L} \times U(3)_{R}\right] /\left[U(2)_{L} \times U(2)_{R}\right]$ breaking, the $c_{\mathrm{ns}}$ leads to $\left[U(2)_{L} \otimes U(2)_{R}\right] / U(2)_{I}$ breaking. Furthermore, the linear $\sigma$ term in Eq. (13) induces $\sigma$-vacuum transitions which supply the scalar fields with non-zero vacuum expectation values (v.e.v) (hereafter denoted by $\langle\cdots\rangle)$. To simplify notations, let us re-denote $\langle\sigma\rangle$ by $V$ with $V=\operatorname{diag}(a, a, b)$, where $a$ and $b$ denote the vacuum expectation values of the strange and non-strange quarkonium, respectively,

$$
a=\frac{1}{\sqrt{2}}\left\langle\sigma_{\mathrm{ns}}\right\rangle, \quad b=\left\langle\sigma_{\mathrm{s}}\right\rangle
$$

We now shift, as usual, the old $\sigma$ field to a new scalar field $S=\sigma-V$ such that $\langle S\rangle=0$. In this way, new mass terms, three-meson interactions, and a linear term are generated. In particular, the mass and linear terms read

$$
\begin{aligned}
\mathcal{L}_{2}= & -\frac{1}{2}\left(\mu^{2}+\lambda^{\prime}\left(2 a^{2}+b^{2}\right)-2 \lambda a b\right) \operatorname{tr}\left[S^{2}+P^{2}\right] \\
& -\lambda(a+b) \operatorname{tr}\left[V\left(S^{2}+P^{2}\right)\right]-\frac{\lambda}{2} \operatorname{tr}\left[(V S)^{2}-(V P)^{2}\right] \\
& +\beta(2 a+b) \operatorname{tr}\left[S^{2}-P^{2}\right]-2 \beta \operatorname{tr}\left[V\left(S^{2}-P^{2}\right)\right] \\
& -\beta(2 a+b)\left\{(\operatorname{tr}[S])^{2}-(\operatorname{tr}[P])^{2}\right\}+2 \beta(\operatorname{tr}[S] \operatorname{tr}[V S]-\operatorname{tr}[P] \operatorname{tr}[V P]) \\
& -\lambda^{\prime}(\operatorname{tr}[V S])^{2}, \\
\mathcal{L}_{1}= & \operatorname{tr}[c S]-\left\{\mu^{2}+\lambda^{\prime}\left(2 a^{2}+b^{2}\right)+\lambda\left(a^{2}+a b+b^{2}\right)-2 \beta a\right\} \operatorname{tr}[S V] \\
& +a(a+b)(\lambda b-2 \beta) \operatorname{tr}[S] .
\end{aligned}
$$

The above mentioned terms are affected - via the 't Hooft determinant (see the terms proportional to the parameter $\beta$ ) - by the $U_{A}(1)$ anomaly which couples to the v.e.v's of the scalar fields by the spontaneous breaking of chiral symmetry. The consequence is the breaking of the original symmetry down to $S U(2)_{I}$ isospin. A detailed analysis of the breaking pattern for different values of the parameters and the corresponding implications for Goldstone modes in this model was carried in [20]. We refer the interested reader to this work for further details.

The masses of the seven unmixed pseudoscalar and scalar mesons corresponding to the original Gell-Mann matrices $\lambda_{i}(i=1, \ldots, 7)$, namely the isovector pseudoscalar $(\pi)$ and scalar $\left(a_{0}\right)$ mesons as well as the two isodoublets of pseudoscalar $(K)$ and scalar $(\kappa)$ mesons, are obtained from the first five terms of 
the second-order lagrangian (15) as [21-23]

$$
\begin{array}{ll}
m_{\pi}^{2}=\xi+2 \beta b+\lambda a^{2}, & m_{a_{0}}^{2}=\xi-2 \beta b+3 \lambda a^{2}, \\
m_{K}^{2}=\xi+2 \beta a+\lambda\left(a^{2}-a b+b^{2}\right), & m_{\kappa}^{2}=\xi-2 \beta a+\lambda\left(a^{2}+a b+b^{2}\right),
\end{array}
$$

where we used the convenient short-hand notation ${ }^{2} \xi \equiv \mu^{2}+\lambda^{\prime}\left(2 a^{2}+b^{2}\right)$. The elimination of the linear terms of the first-order lagrangian (16) imposes the following constraints on the explicit-symmetry-breaking terms $c_{\mathrm{ns}}$, and $c_{\mathrm{s}}$ :

$$
c_{\mathrm{ns}}=\sqrt{2} a m_{\pi}^{2}, \quad c_{\mathrm{s}}=b m_{K}^{2}+a\left(m_{K}^{2}-m_{\pi}^{2}\right)
$$

In Ref.[22] the PCAC relations for the pion and kaon field are discussed. Whereas the PCAC relation of the pion can be directly read off the $c_{\mathrm{ns}}$ term, the one of the kaon has to be inferred from the linear combination $\left(c_{\mathrm{s}}+c_{\mathrm{ns}} / \sqrt{2}\right) / \sqrt{2}$ :

$$
f_{\pi}=\sqrt{2} a, \quad f_{K}=\frac{1}{\sqrt{2}}(a+b) .
$$

The mass term of the lagrangian involving the mixed isoscalar pseudoscalar and scalar fields, which correspond to the unconventional $\lambda_{\mathrm{ns}}$ and $\lambda_{\mathrm{s}}$ matrices, gets in addition to the contributions from the first five terms also contributions from the last three terms of (15) and reads therefore

$$
\begin{aligned}
\mathcal{L}_{\text {mass }}= & -\frac{1}{2}\left(m_{P_{\mathrm{ns}}}^{2} P_{\mathrm{ns}}^{2}+m_{P_{\mathrm{s}}}^{2} P_{\mathrm{s}}^{2}+2 m_{P_{\mathrm{s}-\mathrm{ns}}}^{2} P_{\mathrm{s}} P_{\mathrm{ns}}\right) \\
& -\frac{1}{2}\left(m_{S_{\mathrm{ns}}}^{2} S_{\mathrm{ns}}^{2}+m_{S_{\mathrm{s}}}^{2} S_{\mathrm{s}}^{2}+2 m_{S_{\mathrm{s}-\mathrm{ns}}}^{2} S_{\mathrm{s}} S_{\mathrm{ns}}\right)
\end{aligned}
$$

with

$$
\begin{aligned}
m_{P_{\mathrm{ns}}}^{2}=\xi-2 \beta b+\lambda a^{2}, & m_{S_{\mathrm{ns}}}^{2}=\xi+2 \beta b+3 \lambda a^{2}+4 \lambda^{\prime} a^{2} \\
m_{P_{\mathrm{s}}}^{2}=\xi+\lambda b^{2}, & m_{S_{\mathrm{s}}}^{2}=\xi+3 \lambda b^{2}+2 \lambda^{\prime} b^{2} \\
m_{P_{\mathrm{s}-\mathrm{ns}}}^{2}=-2 \sqrt{2} \beta a, & m_{S_{\mathrm{s}-\mathrm{ns}}}^{2}=2 \sqrt{2}\left(\beta+\lambda^{\prime} b\right) a .
\end{aligned}
$$

Here, $m_{\chi_{\mathrm{s}}}$ and $m_{\chi_{\mathrm{ns}}}$ with $\chi \in\{P, S\}$ are the masses of the strange and non-strange (pseudo-)scalar quarkonia respectively, while $m_{\chi_{s-n s}}^{2}$, which does not need to be positive, denotes the transition mass-matrix elements of the strange-non-strange (pseudo-)scalar quarkonia. Equations (23) show that the

$\overline{2}$ In the pseudoscalar sector, the "bare mass" $\mu$ solely appears inside this combination. 
mixing between strange and non-strange quarkonia is due to the instantoninduced interactions and the spontaneous breakdown of chiral symmetry.

In the following we will first discuss the mixed pseudoscalar sector. The physical isoscalar pseudoscalar fields are linear combinations of $P_{\mathrm{s}}, P_{\mathrm{ns}}$ which diagonalize the pseudoscalar part of $\mathcal{L}_{\text {mass }}$ :

$$
\begin{gathered}
\eta=P_{\mathrm{ns}} \cos \phi_{P}-P_{\mathrm{s}} \sin \phi_{P} \\
\eta^{\prime}=P_{\mathrm{ns}} \sin \phi_{P}+P_{\mathrm{s}} \cos \phi_{P}
\end{gathered}
$$

This diagonalization of the mass matrix for the pseudoscalar mesons yields the relations

$$
\sin 2 \phi_{P}=\frac{2 m_{P_{\mathrm{s}-\mathrm{ns}}}^{2}}{m_{\eta^{\prime}}^{2}-m_{\eta}^{2}}, \quad \cos 2 \phi_{P}=\frac{m_{P_{\mathrm{s}}}^{2}-m_{P_{\mathrm{ns}}}^{2}}{m_{\eta^{\prime}}^{2}-m_{\eta}^{2}} .
$$

Here, $\phi_{P}$ stands for the isoscalar-pseudoscalar mixing angle as introduced in Eq. (3) above. In addition, one finds the following trace relation

$$
m_{\eta^{\prime}}^{2}+m_{\eta}^{2}=m_{P_{\mathrm{s}}}^{2}+m_{P_{\mathrm{ns}}}^{2}
$$

to be valid. As a trivial consequence of Eq. (25), the following relation holds: $\left(m_{\eta^{\prime}}^{2}-m_{\eta}^{2}\right)^{2}=4 m_{P_{\mathrm{s}-\mathrm{ns}}}^{4}+\left(m_{P_{\mathrm{s}}}^{2}-m_{P_{\mathrm{ns}}}^{2}\right)^{2}$. Together with Eq. (26) it induces

$$
m_{\eta^{\prime}}^{2} m_{\eta}^{2}=m_{P_{\mathrm{s}}}^{2} m_{P_{\mathrm{ns}}}^{2}-\left(m_{P_{\mathrm{s}-\mathrm{ns}}}^{2}\right)^{2}
$$

such that

$$
m_{\eta / \eta^{\prime}}^{2}=\frac{1}{2}\left(m_{P_{\mathrm{s}}}^{2}+m_{P_{\mathrm{ns}}}^{2}\right) \mp \sqrt{\frac{1}{4}\left(m_{P_{\mathrm{s}}}^{2}-m_{P_{\mathrm{ns}}}^{2}\right)^{2}+\left(m_{P_{\mathrm{s}-\mathrm{ns}}}^{2}\right)^{2}} .
$$

The five parameters entering the pseudoscalar sector of the model $(\xi, \lambda, \beta, a, b)$ can be fixed through the masses and the decay constants of the pseudoscalars $\left(m_{\eta^{\prime}}, m_{\eta}, m_{\pi}, m_{K}, f_{\pi}\right)$ and can be used to predict all the other properties of the pseudoscalar mesons such as the mixing of the strange and non-strange fields (see model 1 of Table 1). Alternatively, the kaon decay constant $f_{K}$ can be used as input, replacing the combination $\left(m_{\eta^{\prime}}^{2}-m_{\eta}^{2}\right)$ of the above given quantities [23] (see model 2 of Table 1 with the input $\left.\left(\sqrt{m_{\eta^{\prime}}^{2}+m_{\eta}^{2}}, m_{\pi}, m_{K}, f_{\pi}, f_{K}\right)\right)$. The latter procedure creates slightly different results for the pseudoscalar mixing angle. Finally, one could also have used the pseudoscalar mixing angle as input [21] (see models $3 \mathrm{a}$ and $3 \mathrm{~b}$ of Table 1 with the input $\left.\left(m_{\eta}, m_{\eta^{\prime}}, m_{\pi}, \theta_{P}, f_{\pi}\right)\right)$. This leads to a different identification 
of the scalar nonet. The pertinent masses turn out to be highly sensitive to the choice for the input parameters. In particular, the latter version yields heavy scalars [21].

Now, by inserting (17) for $m_{\pi}^{2}, m_{K}^{2}$ and (21-23) for $m_{P_{\mathrm{ns}}}^{2}, m_{P_{\mathrm{s}}}^{2}, m_{P_{\mathrm{s}-\mathrm{ns}}}^{2}$ where the latter are linked to $m_{\eta}^{2}, m_{\eta^{\prime}}^{2}$ by the relations (26-27), one can express the $\beta$ parameter in terms of the pseudoscalar meson masses according to

$$
-4 \beta(a+b)=\frac{\left(m_{\eta^{\prime}}^{2}-m_{\pi}^{2}\right)\left(m_{\eta}^{2}-m_{\pi}^{2}\right)}{\left(m_{K}^{2}-m_{\pi}^{2}\right)} .
$$

The physical solution found here coincides with the phenomenologically favored one of the two solutions of the quadratic equation for $\beta$ reported in an earlier work [22]. The parameter $a$ can be directly fixed through the first of Eqs. (19), whereas $b$, parameterized as $b=(1+2 x) a$, can be fixed either through a fit of the kaon mass as $x=x_{N}=0.37$ [22], or directly through $f_{K}$ in the second of Eqs. (19) as $x=x_{T}=0.22$ [23]. Using, e.g., $x=0.37$ we obtain $\beta \approx-1.55 \mathrm{GeV}$ from (29) and

$$
\sin 2 \phi_{P}=0.9202, \quad \cos 2 \phi_{P}=-0.3911
$$

from the mixing relations (25) together with Eq. (28) and $m_{P_{\mathrm{s}}}^{2}<m_{P_{\mathrm{ns}}}^{2}$ (see Table 1). A careful analysis of the mass matrix shows that the actual mixing angle in the flavor basis is the one arising from the cosine relation in Eq. (30). This angle is complementary to the one arising from the sine relation, which does not distinguish between $\pi / 2-\phi_{P}$ and $\phi_{P}$. The mixing angle in the flavor basis thus turns out to lie within the range determined by the case $x=0.22$, namely $\phi_{P}=49.7^{\circ}$, on the one hand, and the case $x=0.37$, namely $\phi_{P}=56.0^{\circ}$, on the other hand (see Table 1). The corresponding angle in the singlet-octet basis is $\theta_{P}=\phi_{P}-54.7^{\circ}$, where $54.7^{\circ}$ results from the ideal mixing angle in the ns- $\mathrm{s}$ basis. The so-determined values of $\theta_{P}$ are in the range $\theta_{P} \in\left[-5^{\circ},+2^{\circ}\right]$ and therefore close to zero This finding, in combination with the fact that the sole mixing mechanism of flavor fields is the instanton-induced interaction (see the left Eq. (23)), establishes the main result of this section: 't Hooft's instantoninduced interaction mixes strange and non-strange pseudoscalar fields in such a way that one of the physical fields becomes a member of the octet, while the other one becomes an U(3) singlet.

The fact that models $3 \mathrm{a}$ and $3 \mathrm{~b}$ have a non-zero mixing angle is in no contradiction to this as the empirical value(s) of the mixing angle is used as one of the input parameters. As discussed below, the small deviation from the value zero (in comparison to the size of the ideal mixing) should be traced back to subleading contributions, as e.g. mesonic loops.

If the coefficient $\lambda^{\prime}$ in Eq. (23) is ignored, the mixing between the scalar strange 
and non-strange quarkonia due to 't Hooft's instanton-induced interaction is predicted to be of the same size as the corresponding mixing in the pseudoscalar sector but with opposite sign. This is consistent with the results in $[24,25]$. In the scalar sector, however, one has to account for the additional effect brought about by one of the chiral invariants in Eq. (6) whose strength is measured by the above-mentioned $\lambda^{\prime}$ coupling as dictated by the right relation of (23). As discussed in [23], this chiral invariant corresponds to OZI-rule violating disconnected hairpin diagrams. They represent one out of various examples of subleading OZI-rule violating mechanisms, the most important among them being probably Lipkin's non-planar hadronic loops [26]: in the scalar sector, the cancellation of hadronic loops is strongly spoiled by parity conservation $[27,28]$. Thus, although subleading and suppressed with respect to instanton contributions, this effect acquires importance as it interferes destructively with the instanton-induced contribution to the mixing of the scalar mesons. This renders the scalars less strongly mixed than pseudoscalars and thus closer to the flavor basis. Estimates based on meson spectrum and on recent data on radiative $\phi$ decays involving scalars yield for the isoscalar-scalar mixing angle $\phi_{S} \in\left[-9^{\circ},-14^{\circ}\right][18,19,22]$. Therefore one of the scalar isoscalar mesons (sigma) can be nearly identified with the non-strange scalar and is strongly moved down relative to the scalar isovector $\left(a_{0}\right)$ as can be seen from Eqs. $(21,17)$. This is also consistent with results in [24].

It is worth noting that the physical properties of mesons, belonging to sectors which are not affected by the instanton-induced interactions, such as the spin$1^{--}$and tensor $2^{++}$mesons, are well described in terms of almost pure flavor states. The small departure from the flavor basis in these sectors $\left(\phi_{V} \approx 4^{\circ}\right)$ can be attributed to strong and yet incomplete cancellations of all meson loops in this sector $[27,28]$. The same argument can be used for the actual deviation of the $\eta$ from being a pure octet state. The effect is slightly larger in that case due to incomplete cancellations among hadronic loops [26-28].

\section{Instanton dynamics and the $\eta N N$ coupling}

The co-existence of strange and non-strange quarkonia in the wave function of the $\eta$ meson raises the question on the $\eta_{\mathrm{s}}$ creation by the non-strange nucleon 3 . If the OZI rule were unbroken in the pseudoscalar sector, the valence quarks of the non-strange nucleon could not contribute at all. The only possible direct source for the $\eta_{\mathrm{s}} N N$ vertex would be the hidden strangeness of the nucleon, i.e. the existence of small, but non-negligible $(u u d)(\bar{s} s)$ configurations in the proton wave function, see Fig. 1 . The main $\bar{s} s$-source, however, is

3 In this chapter we use the conventional notation $\eta_{\mathrm{ns}}$ and $\eta_{\mathrm{s}}$ for the non-strange and strange pseudoscalar fields instead of the notation $P_{\mathrm{ns}}$ and $P_{\mathrm{s}}$ of Section 2 . 
the conversion of a non-strange quarkonium, emitted by the valence quarks (of the nucleon), into the strange quarkonium under the influence of the OZI-rule violating instanton effects. There seem to exist two mechanisms contributing to this conversion. The first mechanism is displayed in Fig. 2 and has to do with mass terms generated by the anomaly when spontaneous breaking of chiral symmetry takes place. In this case a $\bar{q} q$ pair is replaced by its v.e.v and the flavor eigenstates $\eta_{\mathrm{s}}$ and $\eta_{\mathrm{ns}}$ get mixed - an effect which has been quantified in Section 2 in the context of the broken linear sigma model. The second mechanism, displayed in Fig. 3, involves a direct instanton-induced interaction. Finally, less important sources for $(\bar{s} s)$ quarkonia are non-planar kaon loop diagrams of the type presented in Fig. 4.

In addition to the $\eta_{\mathrm{s}}$ production mechanisms mentioned above there exist also contributions from $\eta_{\text {ns }}$ production to the $\eta N N$ interaction. These contain the conventional mechanisms with continuous quark lines in addition to instantoninduced interactions involving the hidden strangeness of the nucleon. Clearly, the description of the $\eta N N$ interaction requires the disentanglement and quantification of all these mechanisms. This can be accomplished in a natural way in the framework of the model discussed in Section 2. To this end, we need to study the production of flavor fields from the flavor axial currents which we discuss in the framework of the same model.

The strange and non-strange weak decay constants are defined in the usual way:

$$
\left\langle 0\left|A_{\mu}^{\mathrm{ns}}(0)\right| \eta_{\mathrm{ns}}(q)\right\rangle=i f_{\eta_{\mathrm{ns}}} q_{\mu}, \quad\left\langle 0\left|A_{\mu}^{\mathrm{s}}(0)\right| \eta_{\mathrm{s}}(q)\right\rangle=i f_{\eta_{\mathrm{s}}} q_{\mu}
$$

Under the axial transformations

$$
\delta_{A} M=-(i / \sqrt{2})\left\{\epsilon^{A}, M\right\}, \quad \delta_{A} M^{\dagger}=(i / \sqrt{2})\left\{\epsilon^{A}, M^{\dagger}\right\}
$$

with $\epsilon^{A}=\frac{1}{\sqrt{2}} \lambda_{i} \epsilon_{i}^{A}$, the lagrangian in Eq. (5) is not any longer invariant because of the explicit and instanton-induced symmetry breaking terms. A calculation of the divergences of the strange and non-strange axial currents in the model yields:

$$
\partial^{\mu} A_{\mu}^{\mathrm{ns}}=c_{\mathrm{ns}} \eta_{\mathrm{ns}}+2 \beta W, \quad \partial^{\mu} A_{\mu}^{\mathrm{s}}=\sqrt{2} c_{\mathrm{s}} \eta_{\mathrm{s}}+\sqrt{2} \beta W
$$

where $\mathrm{W}$ stands for the contribution of the instantons and contains trilinear, bilinear and linear terms in the fields. Explicitly

$$
\begin{aligned}
W & =i\left(\operatorname{det}(M)-\operatorname{det} M^{\dagger}\right) \\
& =-2 \sqrt{2} a b \eta_{\mathrm{ns}}-2 a^{2} \eta_{\mathrm{s}}+\text { bilinear }+ \text { trilinear } .
\end{aligned}
$$


Taking the derivative of both Eqs. (31) and then inserting (33) and (34), we obtain

$$
f_{\eta_{\mathrm{ns}}} m_{\eta_{\mathrm{ns}}}^{2}=c_{\mathrm{ns}}-4 \sqrt{2} \beta a b, \quad f_{\eta_{\mathrm{s}}} m_{\eta_{\mathrm{s}}}^{2}=\sqrt{2} c_{\mathrm{s}}-2 \sqrt{2} \beta a^{2}
$$

Notice that in the case when the anomaly is absent, the masses of both the strange and non-strange fields (which in this fictitious case are the physical fields) are solely driven by the explicit breaking terms (quark mass terms). Therefore these fields are genuine pseudo-Goldstone boson fields in this case. This property is spoiled by the anomaly for both fields as can also be seen from the mass relations

$$
m_{\eta_{\mathrm{ns}}}^{2}-m_{\pi}^{2}=-4 \beta b, \quad b m_{\eta_{\mathrm{s}}}^{2}+2 \beta a^{2}=c_{\mathrm{s}}
$$

We will come back to this point below. But first let us insert the relations (36) in Eq. (35), such that the non-strange and strange weak decay constants are determined as

$$
f_{\eta_{\mathrm{ns}}}=\sqrt{2} a=f_{\pi}, \quad f_{\eta_{\mathrm{s}}}=\sqrt{2} b=2 f_{K}-f_{\pi}
$$

These predictions (see Table 1 for the results of models $1-3 \mathrm{~b}$ ) cannot not be directly compared with the values $f_{\mathrm{q}}=(99 \pm 2) \mathrm{MeV}$ and $f_{\mathrm{s}}=(124 \pm 6) \mathrm{MeV}$ from Ref. [10], since the definitions are different, especially for $f_{\mathrm{s}}$ and our $f_{\eta_{\mathrm{s}}}$.

In order to clarify whether the (octet-) eta is a pseudo-Goldstone boson or not, let us discuss the fictitious case that the explicit breaking (18) in the non-strange sector, $c_{\mathrm{ns}}$, is put by hand to zero, but the one in the strange sector is kept finite, $c_{\mathrm{s}} \neq 0$. Then only the pion remains a Goldstone-boson. The kaon, however, behaves as a pseudo-Goldstone boson, since in this case its squared mass is proportional to the explicit breaking $c_{\mathrm{s}} \propto m_{s}$. Moreover, the non-vanishing of the (squared) $K$-meson mass in the $m_{\pi}^{2} \rightarrow 0$ limit, $m_{K}^{2}=$ $(b-a)(\lambda b-2 \beta) \neq 0$, requires that $b \neq a$, i.e. that the v.e.v. generated by the spontaneous breaking in the strange sector differs from the one in the two non-strange sectors. For this case it can be shown from (21-23) and (26-27) that the physical $\eta$ meson has the following squared mass

$$
\begin{aligned}
m_{\eta}^{2} & =-3 \beta b+\frac{1}{2} \lambda\left(b^{2}-a^{2}\right)-\sqrt{\left(8 a^{2}+b^{2}\right) \beta^{2}+\lambda b\left(b^{2}-a^{2}\right) \beta+\frac{1}{4} \lambda^{2}\left(b^{2}-a^{2}\right)^{2}} \\
& \neq 0
\end{aligned}
$$

It is strongly affected by the anomaly since it does not vanish in the case $b \neq a$ unless the anomaly is nullified $(\beta=0)$. In this sense, the $\eta$ meson is not a 
Goldstone boson. But even for finite $\beta<0$ 4 , its mass still vanishes together with the mass of the kaon, when $b \rightarrow a$. Because the v.e.v. $a$ does not need to vanish here, chiral symmetry is spontaneously broken and the $\eta$ meson is then a pseudo-Goldstone boson, although strongly affected by the anomaly. Note that the mass of the $\eta^{\prime}$ meson does not vanish in the last case and therefore the $\eta^{\prime}$ cannot become a pseudo-Goldstone boson for a non-vanishing (negative) $\beta$ and $b \rightarrow a \neq 0$, since in the expression of $m_{\eta^{\prime}}^{2}$ the analogous square root to the one in Eq. (38) shows the opposite sign.

As a starting point in the analysis of the $\eta N N$ coupling we will assume that the anomaly is absent, i.e. the parameter $\beta=0$. In this case the $\eta_{\mathrm{ns}}$ couples to the non-strange nucleon only via the $u, d$ (valence and sea) components of the nucleon, whereas the $\eta_{\mathrm{s}}$-nucleon coupling is solely induced by the hidden $\bar{s} s$ component of the nucleon. The $\eta_{\mathrm{s}}$ and $\eta_{\mathrm{ns}}$ fields are genuine Goldstone bosons in this case, and we can assume - without loss of generality - that their interaction with the nucleon follows the derivative structure (as it has to be the case in the non-linear realization of the pseudoscalar fields in chiral perturbation theory)

$$
\begin{aligned}
\mathcal{L}_{\eta_{\mathrm{ns}} N N} & =\left(\frac{\partial_{\mu} \eta_{\mathrm{ns}}}{f_{\eta_{\mathrm{ns}}}}\right) g_{A}^{\mathrm{ns}} \bar{\Psi}_{N} \gamma^{\mu} \gamma^{5} \frac{1}{2} \Psi_{N} \\
\mathcal{L}_{\eta_{\mathrm{s}} N N} & =\left(\frac{\partial_{\mu} \eta_{\mathrm{s}}}{f_{\eta_{\mathrm{s}}}}\right) g_{A}^{\mathrm{s}} \bar{\Psi}_{N} \gamma^{\mu} \gamma^{5} \frac{1}{2} \Psi_{N}
\end{aligned}
$$

where $g_{A}^{\mathrm{ns}}=a_{u}+a_{d}$ and $g_{A}^{\mathrm{s}}=\sqrt{2} a_{s}$. The quantities $a_{u}, a_{d}$, and $a_{s}$ are the fractions of proton spin carried by the $u, d$, and $s$ quark seas (including valence contributions), respectively, and are known from deep inelastic scattering data [29]. They are parameterized as

$$
\begin{aligned}
a_{q_{i}}\left(Q^{2}\right) & =\Delta q_{i}\left(Q^{2}\right)-\frac{\alpha_{s}\left(Q^{2}\right)}{2 \pi} \Delta g\left(Q^{2}\right), \quad q_{i}=u, d, s \\
Q^{2} & =-\left(p-p^{\prime}\right)^{2}
\end{aligned}
$$

where $\Delta q_{i}\left(Q^{2}\right)$ is the genuine spin fraction associated with the $q_{i}$ flavored quark sea, while the $\Delta g\left(Q^{2}\right)$ term describes de-polarization effects due to gluon contributions. We use below the values of the spin-fractions reported in [29] for $Q^{2}=10 \mathrm{GeV}^{2}$ as $a_{u}=0.82 \pm 0.02, a_{d}=-0.44 \pm 0.02$, and $a_{s}=-0.10 \pm 0.02$.

In Eqs. $(39,40)$ it is assumed that the $\eta_{\mathrm{ns}}$ and $\eta_{\mathrm{s}}$ are the flavor-eigenstates of a pseudo-vectorial $\eta$-nucleon interaction. For this reason, the interactions (39) and (40) are designed as the non-strange and strange equivalents of the

4 Remember that $\beta$ has to be negative semi-definite as otherwise the squared $\eta$ and $\eta^{\prime}$ masses are not positive semi-definite. 
standard $\pi N$ Goldberger-Treiman coupling, respectively. In the spirit of an effective chiral expansion, $\mathcal{L}_{\eta_{\mathrm{ns}} N N}$ and $\mathcal{L}_{\eta_{\mathrm{s}} N N}$ would give the leading axial couplings in these two sectors. The latter would be a sensible approximation if $\eta_{\mathrm{ns}}$ and $\eta_{\mathrm{s}}$ could be treated as Goldstone bosons. However, as our discussion in Section 2 showed, both states are strongly affected by the anomaly, and therefore we must be careful about this point.

Let us first focus on the effects due to the (anomaly-induced) mixing of flavor fields during propagation. The calculation of these contributions to the $\eta N N$ coupling can be formulated in a coupled-channel scheme. In this formalism the eta-state can be written as a vector in the $\eta_{\mathrm{ns}}-\eta_{\mathrm{s}}$ space:

$$
\left(\begin{array}{c}
\cos \phi_{P} \\
-\sin \phi_{P}
\end{array}\right)
$$

(see Eq. (3)). In the case when the anomaly is turned off, this state couples (in its transposed form) via the diagonal 'off-shell' propagator to the axial vector current of the nucleon, i.e.

$$
\left(\cos \phi_{P},-\sin \phi_{p}\right) \times\left(\begin{array}{cc}
\frac{i}{q^{2}-m_{\eta_{\mathrm{ns}}}^{2}} & 0 \\
0 & \frac{i}{q^{2}-m_{\eta_{\mathrm{s}}}^{2}}
\end{array}\right) \times\left(\begin{array}{c}
\frac{g_{A}^{\mathrm{ns}}}{f_{\eta_{\mathrm{ns}}}} \\
\frac{g_{A}^{\mathrm{s}}}{f_{\eta_{\mathrm{s}}}}
\end{array}\right) i q_{\mu} \bar{\Psi}_{N} \gamma^{\mu} \gamma^{5} \frac{1}{2} \Psi_{N}
$$

In other words, the strange-non-strange fields are the propagating fields.

Let us now consider the physical case when the anomaly is present. As a consequence, one encounters the mixing of the flavor fields during propagation rendering propagating $\eta$ and $\eta^{\prime}$. For this reason, one has to replace the diagonal propagator matrix in Eq. (43) by the full propagator matrix including the anomaly-induced non-diagonal elements :

$$
i\left(\begin{array}{cc}
q^{2}-m_{\eta_{\mathrm{ns}}}^{2} & -m_{\eta_{\mathrm{s}-\mathrm{ns}}}^{2} \\
-m_{\eta_{\mathrm{s}-\mathrm{ns}}}^{2} & q^{2}-m_{\eta_{\mathrm{s}}}^{2}
\end{array}\right)^{-1}=\frac{i}{\left(q^{2}-m_{\eta}^{2}\right)\left(q^{2}-m_{\eta^{\prime}}^{2}\right)}\left(\begin{array}{cc}
q^{2}-m_{\eta_{\mathrm{s}}}^{2} & m_{\eta_{\mathrm{s}-\mathrm{ns}}}^{2} \\
m_{\eta_{\mathrm{s}-\mathrm{ns}}}^{2} & q^{2}-m_{\eta_{\mathrm{ns}}}^{2}
\end{array}\right)
$$

With the aid of the following relations which arise from the diagonalization of the pseudoscalar isoscalar sector

$$
\begin{gathered}
m_{\eta_{\mathrm{s}}}^{2} \cos \phi_{P}+m_{\eta_{\mathrm{s}-\mathrm{ns}}}^{2} \sin \phi_{P}=m_{\eta^{\prime}}^{2} \cos \phi_{P} \\
m_{\eta_{\mathrm{ns}}}^{2} \sin \phi_{P}+m_{\eta_{\mathrm{s}-\mathrm{ns}}}^{2} \cos \phi_{P}=m_{\eta^{\prime}}^{2} \sin \phi_{P}
\end{gathered}
$$




$$
\begin{gathered}
m_{\eta_{\mathrm{s}}}^{2} \sin \phi_{P}-m_{\eta_{\mathrm{s}-\mathrm{ns}}}^{2} \cos \phi_{P}=m_{\eta}^{2} \sin \phi_{P} \\
m_{\eta_{\mathrm{ns}}}^{2} \cos \phi_{P}-m_{\eta_{\mathrm{s}-\mathrm{ns}}}^{2} \sin \phi_{P}=m_{\eta}^{2} \cos \phi_{P}
\end{gathered}
$$

one can cast Eq. (43), where the diagonal propagator matrix is now replaced by the full propagator matrix Eq. (44), into the following form:

$$
\frac{i}{q^{2}-m_{\eta}^{2}}\left(\frac{g_{A}^{\mathrm{ns}}}{f_{\eta_{\mathrm{ns}}}} \cos \phi_{P}-\frac{g_{A}^{\mathrm{s}}}{f_{\eta_{\mathrm{s}}}} \sin \phi_{P}\right) i q_{\mu} \bar{\Psi}_{N} \gamma^{\mu} \gamma^{5} \frac{1}{2} \Psi_{N} .
$$

This last equation illustrates the formation of propagating $\eta$ and (by a similar calculation) $\eta^{\prime}$ fields under the influence of anomaly-induced mixing effects during the propagating stage of the isoscalar pseudoscalars. The coupling strengths of the $\eta$ and $\eta^{\prime}$ fields to the nucleon are determined as

$$
g_{\eta N N}=\frac{g_{A}^{\mathrm{ns}} M_{N}}{f_{\eta_{\mathrm{ns}}}} \cos \phi_{P}-\frac{g_{A}^{\mathrm{s}} M_{N}}{f_{\eta_{\mathrm{s}}}} \sin \phi_{P}
$$

and

$$
g_{\eta, N N}=\frac{g_{A}^{\mathrm{ns}} M_{N}}{f_{\eta_{\mathrm{ns}}}} \sin \phi_{P}+\frac{g_{A}^{\mathrm{s}} M_{N}}{f_{\eta_{\mathrm{s}}}} \cos \phi_{P}
$$

respectively. Eqs. $(47,48)$ become exactly the octet and singlet relations for the case that $\phi_{P}=\phi_{\mathrm{su}(3)}$ (i.e. $\cos \phi_{P}=\sqrt{1 / 3}$ and $\sin \phi_{P}=\sqrt{2 / 3}$ ) and that $f_{\eta_{\mathrm{ns}}}=f_{\eta_{8}}=f_{\eta_{\mathrm{s}}}=f_{\eta_{0}}=f_{K}=f_{\pi}$ :

$$
g_{\eta N N}=\sqrt{\frac{1}{3}} \frac{M_{N}}{f_{\pi}} a_{8}, \quad g_{\eta^{\prime} N N}=\sqrt{\frac{2}{3}} \frac{M_{N}}{f_{\pi}} a_{0},
$$

where $a_{8}=a_{u}+a_{d}-2 a_{\mathrm{s}}, a_{0}=a_{u}+a_{d}+a_{\mathrm{s}}$.

The expressions (49) for $g_{\eta N N}$ and $g_{\eta^{\prime} N N}$ are the well known octet- and the singlet Goldberger-Treiman relations, respectively. In the literature, as discussed in Section 3.11 of Ref. [10], there exists an other alternative for the description of the Goldberger-Treiman relation in the singlet channel. Shore and Veneziano [30] established a two-component description of the singlet axial charge where the singlet Goldberger-Treiman relation is modified by an additional direct coupling of the pseudoscalar operator $G \widetilde{G}$ to the nucleon,

$$
\sqrt{\frac{2}{3}} \frac{M_{N}}{\widetilde{f}} a_{0}=g_{\eta^{\prime} N N}+\sqrt{\frac{2}{3}} \tilde{f} m_{\eta^{\prime}}^{2} g_{\widetilde{G} N N}
$$


Here $G$ stands for the gluon field, $\widetilde{G}$ for its dual, and the new couplings $g_{\eta^{\prime} N N}$ and $g_{\widetilde{G} N N}$ are related to the polarized quark distributions and polarized gluon distribution, respectively. As it is stressed in Ref. [10], the quantity $\tilde{f}$ does not coincide with the decay constant $f_{\eta_{0}}$. The latter scales as $a_{0}$, whereas $\tilde{f}$ is scale-independent. We refer the reader to Ref. [10] for further details and a comparison of both schemes.

The above considerations could be criticized on two accounts $\square$. First, they are based on special choice of couplings of the flavor-eigenstates $\eta_{\mathrm{ns}}$ and $\eta_{\mathrm{s}}$ to the nucleon. Secondly, the role of the anomaly in the $\eta, \eta^{\prime}$ production at the $\eta, \eta^{\prime}$-nucleon vertex is not explicit. Below, we will close these gaps in the derivation by adapting the usual derivation of the Goldberger-Treiman relation for pions to the $A_{\mathrm{ns}}^{\mu}$ and $A_{\mathrm{s}}^{\mu}$ cases and keeping track of the modifications caused by the $U_{A}(1)$ anomaly. The divergences of these currents (see Eq. (33)), when sandwiched between nucleon states, clearly exhibit some of the anomaly effects in the $\eta\left(\eta^{\prime}\right) N N$ interaction.

On the basis of symmetries (Lorentz covariance, parity etc.), the matrix elements of the flavor currents between nucleon states can be parameterized as follows

$$
\begin{aligned}
\left\langle N^{\prime}\left|A_{\mu}^{\mathrm{ns}}\right| N\right\rangle & =\bar{u}\left(p^{\prime}, s^{\prime}\right)\left[\gamma_{\mu} \gamma_{5} G_{A}^{\mathrm{ns}}\left(q^{2}\right)+q_{\mu} \gamma_{5} G_{P}^{\mathrm{ns}}\left(q^{2}\right)\right] \frac{1}{2} u(p, s), \\
\left\langle N^{\prime}\left|A_{\mu}^{\mathrm{s}}\right| N\right\rangle & =\bar{u}\left(p^{\prime}, s^{\prime}\right)\left[\gamma_{\mu} \gamma_{5} G_{A}^{\mathrm{s}}\left(q^{2}\right)+q_{\mu} \gamma_{5} G_{P}^{\mathrm{s}}\left(q^{2}\right)\right] \frac{1}{2} u(p, s),
\end{aligned}
$$

where $q=\left(p^{\prime}-p\right)$ is the transferred momentum, $s$ and $s^{\prime}$ denote nucleon polarizations, and $G_{A}^{\mathrm{ns}, \mathrm{s}}\left(q^{2}\right)$ and $G_{P}^{\mathrm{ns}, \mathrm{s}}\left(q^{2}\right)$ are the axial vector and induced pseudoscalar form factors, respectively. These form factors can not be fixed on symmetry grounds alone. The divergence of the above matrix elements yields

$$
\begin{aligned}
\left\langle N^{\prime}\left|i \partial^{\mu} A_{\mu}^{\mathrm{ns}}\right| N\right\rangle & =-\bar{u}\left(p^{\prime}, s^{\prime}\right)\left[2 M_{N} G_{A}^{\mathrm{ns}}\left(q^{2}\right)+q^{2} G_{P}^{\mathrm{ns}}\left(q^{2}\right)\right] \frac{\gamma_{5}}{2} u(p, s) \\
& =i\left\langle N^{\prime}\left|\mathrm{c}_{\mathrm{ns}} \eta_{\mathrm{ns}}+2 \beta W\right| N\right\rangle \\
\left\langle N^{\prime}\left|i \partial^{\mu} A_{\mu}^{\mathrm{s}}\right| N\right\rangle & =-\bar{u}\left(p^{\prime}, s^{\prime}\right)\left[2 M_{N} G_{A}^{\mathrm{s}}\left(q^{2}\right)+q^{2} G_{P}^{\mathrm{s}}\left(q^{2}\right)\right] \frac{\gamma_{5}}{2} u(p, s) \\
& =i\left\langle N^{\prime}\left|\sqrt{2} \mathrm{c}_{\mathrm{s}} \eta_{\mathrm{s}}+\sqrt{2} \beta W\right| N\right\rangle .
\end{aligned}
$$

Here use has been made of Eqs. (33). In combining Eqs. (34) and (35) one arrives at

5 Of course, the omission of direct instanton-induced couplings of the type displayed in Fig. 3 and the neglect of subleading non-planar $\bar{K}^{(*)} K^{(*)}$ loops, see Fig. 4 , are further points open to criticism, but outside the scope of the model. 


$$
\begin{gathered}
c_{\mathrm{ns}} \eta_{\mathrm{ns}}+2 \beta W=f_{\eta_{\mathrm{ns}}} m_{\eta_{\mathrm{ns}}}^{2} \eta_{\mathrm{ns}}-4 \beta a^{2} \eta_{\mathrm{s}}+\text { bilinear }+ \text { trilinear } \\
\sqrt{2} \mathrm{c}_{\mathrm{s}} \eta_{\mathrm{s}}+\sqrt{2} \beta W=f_{\eta_{\mathrm{s}}} m_{\eta_{\mathrm{s}}}^{2} \eta_{\mathrm{s}}-4 \beta a b \eta_{\mathrm{ns}}+\text { bilinear }+ \text { trilinear }
\end{gathered}
$$

At low energies, the matrix elements of the pseudoscalar flavor fields between nucleons are dominated by the exchange of propagating $\eta$ and $\eta^{\prime}$ mesons

$$
\begin{aligned}
i\left\langle N^{\prime}\left|\eta_{\mathrm{ns}}\right| N\right\rangle & =\left(\frac{g_{\eta N N}}{q^{2}-m_{\eta}^{2}}\left\langle\eta_{\mathrm{ns}} \mid \eta\right\rangle+\frac{g_{\eta^{\prime} N N}}{q^{2}-m_{\eta^{\prime}}^{2}}\left\langle\eta_{\mathrm{ns}} \mid \eta^{\prime}\right\rangle\right) \bar{u}\left(p^{\prime}, s^{\prime}\right) \gamma_{5} u(p, s) \\
i\left\langle N^{\prime}\left|\eta_{\mathrm{s}}\right| N\right\rangle & =\left(\frac{g_{\eta N N}}{q^{2}-m_{\eta}^{2}}\left\langle\eta_{\mathrm{s}} \mid \eta\right\rangle+\frac{g_{\eta^{\prime} N N}}{q^{2}-m_{\eta^{\prime}}^{2}}\left\langle\eta_{\mathrm{s}} \mid \eta^{\prime}\right\rangle\right) \bar{u}\left(p^{\prime}, s^{\prime}\right) \gamma_{5} u(p, s)
\end{aligned}
$$

In deriving Eqs. (54) we used the following $\eta\left(\eta^{\prime}\right) N N$ lagrangian

$$
\mathcal{L}=g_{\eta N N} \eta \bar{\Psi}_{N} i \gamma_{5} \Psi_{N}+g_{\eta^{\prime} N N} \eta^{\prime} \bar{\Psi}_{N} i \gamma_{5} \Psi_{N}
$$

Note that we now apply couplings of pseudoscalar nature, in contrast to the former derivation which was based on couplings of derivative nature, see Eqs. (39) and (40). Inserting (53,54) in Eqs. (52) and disregarding contributions from bilinear and trilinear terms in the anomaly leads to

$$
\begin{aligned}
\frac{1}{2} G_{P}^{\mathrm{ns}}\left(q^{2}\right) & =\frac{-M_{N} G_{A}^{\mathrm{ns}}\left(q^{2}\right)+g_{\eta N N} F_{\eta}^{\mathrm{ns}}+g_{\eta^{\prime} N N} F_{\eta^{\prime}}^{\mathrm{ns}}}{q^{2}}-\frac{g_{\eta N N} F_{\eta}^{\mathrm{ns}}}{q^{2}-m_{\eta}^{2}}-\frac{g_{\eta^{\prime} N N} F_{\eta^{\prime}}^{\mathrm{ns}}}{q^{2}-m_{\eta^{\prime}}^{2}} \\
\frac{1}{2} G_{P}^{\mathrm{s}}\left(q^{2}\right) & =\frac{-M_{N} G_{A}^{\mathrm{s}}\left(q^{2}\right)+g_{\eta N N} F_{\eta}^{\mathrm{s}}+g_{\eta^{\prime} N N} F_{\eta^{\prime}}^{\mathrm{s}}}{q^{2}}-\frac{g_{\eta N N} F_{\eta}^{\mathrm{s}}}{q^{2}-m_{\eta}^{2}}-\frac{g_{\eta^{\prime} N N} F_{\eta^{\prime}}^{\mathrm{s}}}{q^{2}-m_{\eta^{\prime}}^{2}}
\end{aligned}
$$

Here, we used the definitions

$$
\begin{aligned}
& F_{\eta}^{\mathrm{ns}} \equiv \frac{1}{m_{\eta}^{2}}\left(f_{\eta_{\mathrm{ns}}} m_{\eta_{\mathrm{ns}}}^{2} \cos \phi_{P}+4 \beta a^{2} \sin \phi_{P}\right)=f_{\eta_{\mathrm{ns}}} \cos \phi_{P}, \\
& F_{\eta^{\prime}}^{\mathrm{ns}} \equiv \frac{1}{m_{\eta^{\prime}}^{2}}\left(f_{\eta_{\mathrm{ns}}} m_{\eta_{\mathrm{ns}}}^{2} \sin \phi_{P}-4 \beta a^{2} \cos \phi_{P}\right)=f_{\eta_{\mathrm{ns}}} \sin \phi_{P}, \\
& F_{\eta}^{\mathrm{s}} \equiv \frac{1}{m_{\eta}^{2}}\left(-f_{\eta_{\mathrm{s}}} m_{\eta_{\mathrm{s}}}^{2} \sin \phi_{P}-4 \beta a b \cos \phi_{P}\right)=-f_{\eta_{\mathrm{s}}} \sin \phi_{P}, \\
& F_{\eta^{\prime}}^{\mathrm{s}} \equiv \frac{1}{m_{\eta^{\prime}}^{2}}\left(f_{\eta_{\mathrm{s}}} m_{\eta_{\mathrm{s}}}^{2} \cos \phi_{P}-4 \beta a b \sin \phi_{P}\right)=f_{\eta_{\mathrm{s}}} \cos \phi_{P},
\end{aligned}
$$

where the r.h.s. equations follow from the relations (45), the expressions (37) of $f_{\eta_{\mathrm{ns}}}$ and $f_{\eta_{\mathrm{s}}}$ and $m_{\eta_{\mathrm{s}-\mathrm{ns}}}^{2}$ as defined in (23). Thus the $F_{\eta, \eta^{\prime}}^{\mathrm{ns}, \mathrm{s}}$ are nothing but the weak decay constants for the production of physical $\eta, \eta^{\prime}$ fields by the flavor 
currents (see Eqs. (31)), i.e.

$$
\begin{array}{ll}
\left\langle 0\left|A_{\mu}^{\mathrm{ns}}\right| \eta(q)\right\rangle=i F_{\eta}^{\mathrm{ns}} q_{\mu}, & \left\langle 0\left|A_{\mu}^{\mathrm{ns}}\right| \eta^{\prime}(q)\right\rangle=i F_{\eta^{\prime}}^{\mathrm{ns}} q_{\mu}, \\
\left\langle 0\left|A_{\mu}^{\mathrm{s}}\right| \eta(q)\right\rangle=i F_{\eta}^{\mathrm{s}} q_{\mu}, & \left\langle 0\left|A_{\mu}^{\mathrm{s}}\right| \eta^{\prime}(q)\right\rangle=i F_{\eta^{\prime}}^{\mathrm{s}} q_{\mu},
\end{array}
$$

such that the isoscalar pseudoscalar versions of the PCAC relations are implied (see Eqs. (33) and (35)):

$$
\begin{array}{ll}
\left\langle 0\left|\partial^{\mu} A_{\mu}^{\mathrm{ns}}\right| \eta(q)\right\rangle=F_{\eta}^{\mathrm{ns}} m_{\eta}^{2}, & \left\langle 0\left|\partial^{\mu} A_{\mu}^{\mathrm{ns}}\right| \eta^{\prime}(q)\right\rangle=F_{\eta^{\prime}}^{\mathrm{ns}} m_{\eta^{\prime}}^{2} \\
\left\langle 0\left|\partial^{\mu} A_{\mu}^{\mathrm{s}}\right| \eta(q)\right\rangle=F_{\eta}^{\mathrm{s}} m_{\eta}^{2}, & \left\langle 0\left|\partial^{\mu} A_{\mu}^{\mathrm{s}}\right| \eta^{\prime}(q)\right\rangle=F_{\eta^{\prime}}^{\mathrm{s}} m_{\eta^{\prime}}^{2}
\end{array}
$$

The poles at $q^{2}=0$ in the $G_{P}^{\mathrm{ns}, \mathrm{s}}\left(q^{2}\right)$ form factors in Eq. (56) are unphysical since in the explicitly broken case there exist no massless excitation. These terms can be eliminated, if one requires that the corresponding numerators vanish. This condition leads to the following relations

$$
\begin{aligned}
& M_{N} G_{A}^{\mathrm{ns}}\left(q^{2}\right)=g_{\eta N N} f_{\eta_{\mathrm{ns}}} \cos \phi_{P}+g_{\eta^{\prime} N N} f_{\eta_{\mathrm{ns}}} \sin \phi_{P} \\
& M_{N} G_{A}^{\mathrm{s}}\left(q^{2}\right)=-g_{\eta N N} f_{\eta_{\mathrm{s}}} \sin \phi_{P}+g_{\eta^{\prime} N N} f_{\eta_{\mathrm{s}}} \cos \phi_{P} .
\end{aligned}
$$

Eqs. (60) are the $\eta, \eta^{\prime}$ analogs of the Goldberger-Treiman relation for pions ${ }^{6}$. They are valid for small $q^{2}$ and under the condition that the $G_{A}^{\mathrm{ns}, \mathrm{s}}$ form factors change slowly with $q^{2}$ (they have no poles) in this energy region. One can easily check that Eqs. (60) are compatible with the relations (47) and (48), if at $q^{2}=0$ the axial vector form factors are expressed by the spin fractions $G_{A}^{\mathrm{ns}}(0)=g_{A}^{\mathrm{ns}}=a_{u}+a_{d}, \quad G_{A}^{\mathrm{s}}(0)=g_{A}^{\mathrm{s}}=\sqrt{2} a_{s}$. Thus both derivations are completely consistent: the here discussed contributions of the anomaly to the creation of physical pseudoscalar fields from the vacuum are hidden in the $\sin \phi_{P}$ and $\cos \phi_{P}$ terms (42) in the coupled channel scheme. There is no "direct instanton induced interaction" in this calculation. The place to include such a contribution are the $G_{A}^{\mathrm{s}, \mathrm{ns}}\left(q^{2}\right)$ form factors which here were solely identified with the spin fractions.

The conclusion we extract in this model-dependent analysis of the non-perturbative effects brought about by 't Hooft's effective instanton-induced interaction, is the following: this interaction, in addition to being responsible for the $\eta$ as a member of the pseudoscalar octet, is also responsible for its PCAC behavior and the interactions of the $\eta$ meson with external sources. These

${ }^{6}$ As a cross-check notice that in the case of vanishing $\beta$, the mixing angle $\phi_{P}$ is zero and Eqs. (60) become simply the Goldberger-Treiman relations of the generationflavor fields as postulated in Eqs. $(39,40)$. 
interactions exhibit an octet-like behavior in the case we consider instanton effects during propagation or creation from the vacuum of pseudoscalar fields. Our expectation is that deviations from this behavior are related to the direct instanton induced interactions shown in Fig. 3 and to the inclusion of subleading non-planar $\bar{K}^{(*)} K^{(*)}$ loops.

Using the results and input-values of the models of Table 1 for the quantities involved and the spin-fraction $a_{q_{i}}$ as defined below Eq. (41), we obtain from the re-derived Eqs. (47) and (48)

$$
\begin{aligned}
& g_{\eta N N}=2.8 \pm 0.5 \quad \text { and } \quad g_{\eta^{\prime} N N}=2.8 \pm 0.4 \quad(\text { model } 1) \\
& g_{\eta N N}=3.3 \pm 0.6 \quad \text { and } \quad g_{\eta^{\prime} N N}=2.3 \pm 0.6 \quad(\text { model } 2) \text {, } \\
& g_{\eta N N}=3.5 \pm 0.7 \quad \text { and } \quad g_{\eta^{\prime} N N}=2.0 \pm 0.7 \quad \text { (model 3a) } \\
& g_{\eta N N}=3.9 \pm 0.7 \quad \text { and } \quad g_{\eta^{\prime} N N}=1.2 \pm 0.7 \quad(\text { model } 3 \mathrm{~b}) \text {, }
\end{aligned}
$$

where the errors of $g_{\eta N N}$ and $g_{\eta^{\prime} N N}$ are partially correlated because of the spin fractions and the very precisely determined value of $g_{A}^{3}=a_{u}-a_{d}=$ $1.267 \pm 0.004$, and therefore

$$
\begin{array}{ll}
\frac{g_{\eta N N}^{2}}{4 \pi}+\frac{g_{\eta^{\prime} N N}^{2}}{4 \pi}=1.2 \pm 0.4 & (\text { model } 1), \\
\frac{g_{\eta N N}^{2}}{4 \pi}+\frac{g_{\eta^{\prime} N N}^{2}}{4 \pi}=1.3 \pm 0.4 & \text { (models } 2,3 \mathrm{a}, \text { and } 3 \mathrm{~b})
\end{array}
$$

see Table 1. Note that the displayed error bars have been calculated solely from the errors \pm 0.02 of the spin fractions $a_{q_{i}}$ (which contribute about $70-$ $80 \%$ of the total error), from the error $\pm 4 \mathrm{MeV}$ of $f_{\pi^{0}}$ [8] (which has to be used here instead of the more precisely determined $f_{\pi^{ \pm}}$), and from other uncertainties in the input quantities. Further sources of (systematical) errors could result from the extrapolation from the $q^{2}=0$ point of weak interaction to the mass-shells $m_{\eta}^{2}$ and $m_{\eta^{\prime}}^{2}$ of strong interactions, from higher order corrections in the symmetry breaking, from meson-loop corrections, from the neglect of the subleading OZI-rule-violating disconnected hairpin diagrams (e.g., from an incomplete cancellation of the non-planar $\bar{K}^{(*)} K^{(*)}$ loops), and from the neglect of baryon resonances as e.g. the $S_{11}(1535)$. The values of all four models are still - within the errors - compatible with the upper bound obtained by Grein and Kroll from the analysis of $N N$ forward scattering [31]

$$
\frac{g_{\eta N N}^{2}}{4 \pi}+\frac{g_{\eta^{\prime} N N}^{2}}{4 \pi} \lesssim 1 .
$$

Note that the high-precision measurements of the differential cross sections in $\eta$ photo-production off-proton near threshold at the Mainz Microtron (MAMI) [32] were interpreted in [33] in terms of a strongly suppressed $g_{\eta N N}$ value 
of $\left|g_{\eta N N}\right| \approx 2.25 \pm 0.15$ (or, equivalently $g_{\eta N N}^{2} / 4 \pi \approx 0.4$ ). This result was concluded on the basis of the small $P$-wave contribution to the almost flat angular distributions for a wide range of beam energies. Only our model 1 is compatible with the value of Ref. [33]. From a measurement of $\eta^{\prime}$ production in proton-proton collisions close to threshold at COSY [37] a bound $\left|g_{\eta^{\prime} N N}\right| \leq 2.5$ has been deduced which is compatible with the predictions of all our models within error bars.

The significant suppression of the $\eta N$ coupling relative the octet GoldbergerTreiman relation was noticed by several authors, the most recent being, among others Refs. [34-36]. In Ref. [34] $g_{\eta N N}^{2} / 4 \pi$ was evaluated from QCD sum rules beyond the chiral limit and predicted to range from the still relevant value of $g_{\eta N N}^{2} / 4 \pi=0.42$ (for the $S U(3)$ limit) down to the almost complete decoupling of $g_{\eta N N}^{2} / 4 \pi=0.03$ (beyond the $S U(3)$ limit). Light cone QCD sum rules also lead to the small value of $g_{\eta N N}^{2} / 4 \pi=0.3 \pm 0.15$. Finally, the value of $g_{\eta N N}^{2} / 4 \pi=0.1 \pm 0.01$ was concluded on the basis of recent quark model analyses of meson-photo production data performed in [36]. In the present work, we have revealed the nature of the subtle mechanism of the formation of the octet flavor symmetry under the umbrella of the anomaly as reflected by 't Hooft's effective instanton induced interaction. Our results strongly hint onto the possibility that the reasons for the observed suppression of $g_{\eta N N}$ have to be searched for beyond 't Hooft's effective interaction.

The naive octet-singlet scheme of Eq. (49) predicts $g_{\eta N N}=3.4 \pm 0.6$ and $g_{\eta^{\prime} N N}=2.3 \pm 0.6$ and $1.4 \pm 0.4$ for the Grein-Kroll bound. Note that these results more or less agree with the ones of our model 2. This can be justified from the fact that the mixing angle of this model is the closest to the octetsinglet case, with the exception of model 1 . The latter, however, features a rather large value of $f_{K}$ which severely breaks the condition $f_{K} \approx f_{\pi}$ specified above Eq. (49). Furthermore, the values $g_{\eta N N}=3.4 \pm 0.5$ and $g_{\eta^{\prime} N N}=1.4 \pm 1.1$ of Ref. [10] are calculated with a mixing angle $\phi_{P} \approx 39.3^{\circ}$ which corresponds to $\theta_{P} \approx-15^{\circ}$. Thus this result falls between the ones of model $3 \mathrm{a}$ and $3 \mathrm{~b}$ which are fixed by the mixing angles $\theta_{P}=-10^{\circ}$ and $\theta_{P}=-20^{\circ}$. The rule is that the value of $g_{\eta N N}$ increases and the one of $g_{\eta^{\prime} N N}$ decreases with decreasing mixing angle, while the "Grein-Kroll-strength" is nearly constant (see Eqs. (65) and $(66))$.

\section{Summary}

Admittedly, we have used a rather special model in order to analyze the OZIrule respecting basis of the meson sector and the consequences for the $\eta, \eta^{\prime}$ nucleon coupling constants, namely the chirally symmetric $U(3)_{L} \otimes U(3)_{R}$ linear sigma model. Note, however, that the model-dependence is manifest 
for the scalar sector, whereas the tree-level predictions of the model in the pseudoscalar sector, under the same input of course, ought to be compatible with PCAC arguments or tree-level calculations in chiral perturbation theory, if, and that is the important point, the modifications of the anomaly are properly incorporated into the latter. Furthermore, we have explicitly assumed that the OZI-violation in the isoscalar-pseudoscalar meson sector is governed to leading order by instanton-induced effects rather than by large $N_{c}$ effects, i.e. that meson loops such as the non-planar OZI-violating diagrams, give a non-zero, but subleading contribution to this sector. These points are still controversially discussed in the literature, see e.g. [24] and [38] which favor the instanton interpretation and criticize [25] and [39], respectively (and vice versa), where the large $N_{c}$ interpretation (see also $[10,40]$ ) and the importance of the non-planar meson-loop contributions is advocated for.

However, if we take our assumptions as stated, we find that the mixing of non-strange and strange quarkonia in the wave function of the physical $\eta$ meson induced by the determinantal instanton-induced 't Hooft interaction is such that the $\eta$ meson is close to the octet state. Therefore, the unitary spin symmetry is more obliged for its existence to the effects of the the axial gluon anomaly than being a fundamental symmetry in its own rights. The model also allows us to study consequences for the $\eta N N$ coupling. In two independent calculations, one based on the conventional derivative coupling of the flavoreigenstates to the nucleon, and the other based on a careful study of the axial vector coupling including anomaly contributions (resulting from the 't Hooft interaction) to the nucleon, we obtained the magnitude of $g_{\eta N N}$. We found it, within error bars, to be stable against changes in the input parameters and in addition, to be be close to the ordinary $S U(3)$ results. Though we obtained our $g_{\eta N N}$ to obey a Goldberger-Treiman type relation, the latter did not take its origin from a (massless) pole dominance of the induced pseudoscalar form factor. Rather it appeared as a consequence of the subtle effect of instanton induced flavor-mixing during propagation of the isoscalar pseudoscalars.

In having clarified the role of the axial gluon anomaly (as mimicked by 't Hooft's effective instanton induced interaction) for manufacturing the octet way, we have established the limits beyond which one has to extend the model in order to describe possible deviations of the $g_{\eta N N}$ value from its octet-GTvalue. Among the possible candidates for such effects we emphasize the direct meson-instanton coupling of the type of Fig. 3 and the subleading $\bar{K}^{(*)} K^{(*)}$ loops of the type of Fig. 4.

Our scheme has the advantage that it allows for possible generalizations to non-ideal mixing angles and different values of the meson decay constants in the strange and non-strange sectors, respectively. 


\section{Acknowledgements}

One of us (M.N.) appreciates illuminating correspondence with Professor G. 't Hooft about the extraction of the mixing angle and the sensitivity to the various input-schemes of the model used in Section 2. Work partly supported by CONCyTEG, Mexico under contract 00-16-CONCyTEG-CONACyT-075. 


\section{References}

[1] G. 't Hooft, Phys. Rev. Lett. 37 (1976) 8; Phys. Rev. D14 (1976) 3432.

[2] S. Okubo, Phys. Lett. 5 (1963) 165.

[3] G. Zweig, CERN Report No 8419 TH 412, 1964.

[4] J. Iizuka, Prog. Theor. Phys. Suppl. 37-38 (1966) 21.

[5] M. Kirchbach, Phys. Rev. D58 (1998) 117901.

[6] C. D. Froggatt and H. B. Nielsen, Origin of symmetries, World Scientific, (Singapore, Singapore, 1991).

[7] J. F. Donoghue, E. Golowich and B. R. Holstein, Dynamics of the standard model, Cambridge monographs on particle physics, nuclear physics and cosmology, 2, (Cambridge Univ. Pr., Cambridge, 1992).

[8] The Review of Particle Properties, D. E. Groom et al., Eur. Phys. J. C15 (2000) 1.

[9] T. Feldmann, P. Kroll and B. Stech, Phys. Rev. D58 (1998) 114006, hep$\mathrm{ph} / 9802409$.

[10] T. Feldmann, Int. J. Mod. Phys. A15 (2000) 159, hep-ph/9907491.

[11] G. 't Hooft, Phys. Rept. 142 (1986) 357.

[12] G. Veneziano, Nucl. Phys. B 159, 213 (1979).

[13] E. Witten, Nucl. Phys. B 156, 269 (1979).

[14] C. Rosenzweig, J. Schechter and C. G. Trahern, Phys. Rev. D 21, 3388 (1980).

[15] P. Nath and R. Arnowitt, Phys. Rev. D 23, 473 (1981).

[16] D. Diakonov and M. I. Eides, Problem," Sov. Phys. JETP 54, 232 (1981) [Zh. Eksp. Teor. Fiz. 81, 434 (1981)].

[17] D. Diakonov, hep-ph/9802298.

[18] J. L. Lucio and M. Napsuciale, in: S. Bianco et al. (Eds.), Proc. III Workshop on Physics and Detectors for Daphne (Daphne 99), Frascati Physics Series Vol. XVI, 2000, p. 591, hep-ph/0001136.

[19] J. L. Lucio M. and M. Napsuciale, Phys. Lett. B454 (1999) 365, hep$\mathrm{ph} / 9903234$.

[20] J. T. Lenaghan, D. H. Rischke and J. Schaffner-Bielich, Phys. Rev. D 62, 085008 (2000), nucl-th/0004006.

[21] G. 't Hooft, hep-th/9903189.

[22] M. Napsuciale, hep-ph/9803396. 
[23] N. A. Tornqvist, Eur. Phys. J. C11 (1999) 359, hep-ph/9905282.

[24] T. Schäfer and E. Shuryak, hep-lat/0005025.

[25] N. Isgur and H. B. Thacker, hep-lat/0005006.

[26] H. J. Lipkin, Nucl. Phys. B244 (1984) 147; ibid. B291 (1987) 720.

[27] H. J. Lipkin and B. Zou, Phys. Rev. D53 (1996) 6693.

[28] B. Zou, Phys. Atom. Nucl. 59 (1996) 1427, hep-ph/9611238.

[29] D. Adams et al. [Spin Muon Collaboration (SMC)], Phys. Rev. D56 (1997) 5330, hep-ex/9702005.

[30] G. M. Shore and G. Veneziano, Phys. Lett. B244, (1990) 75; Nucl. Phys. B381 (1992) 23.

[31] W. Grein and P. Kroll, Nucl. Phys. A338 (1980) 332.

[32] B. Krusche et al., Phys. Rev. Lett. 74 (1995) 3736.

[33] L. Tiator, C. Bennhold and S. S. Kamalov, Nucl. Phys. A580 (1994) 455, nuclth/9404013.

[34] H. Kim, T. Doi, and M. Oka, Nucl. Phys. A662, 371 (2000), nucl-th/9909007.

[35] S.-L. Zhu, Phys. Rev. C61, 065205 (2000), nucl-th/0002018.

[36] Q. Zhao, B. Saghai, and Zh. Li, nucl-th/0011069.

[37] P. Moskal et al., Phys. Rev. Lett. 80 (1998) 3202, nucl-ex/9803002; AIP Conf. Proc. 512 (2000) 65, nucl-ex/0007002.

[38] T. DeGrand and A. Hasenfratz, hep-lat/0103002, hep-lat/0012021,

[39] I. Horvath, N. Isgur, J. McCune and H. B. Thacker, hep-lat/0102003.

[40] R. Kaiser and H. Leutwyler, Eur. Phys. J. C 17 (2000) 623, hep-ph/0007101. 


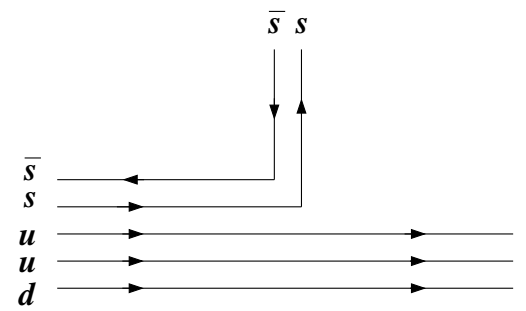

Fig. 1. $\bar{s} s$ production from the hidden strangeness component of the proton. For other notations, see text. (Gluon insertions are suppressed in this and the next two figures.) 


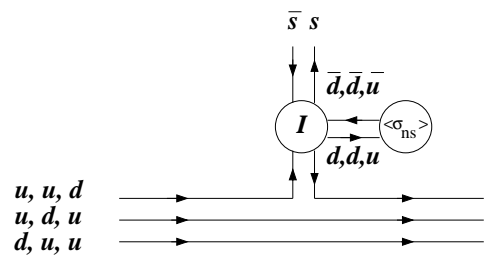

Fig. 2. $\bar{s} s$ production from the valence quarks in the proton due to mass terms generation by the instanton-induced quark interaction coupled to spontaneous breaking of chiral symmetry.

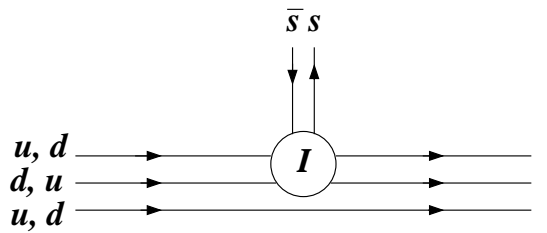

Fig. 3. Instanton-induced direct coupling of $\bar{s} s$ to the nucleon. 


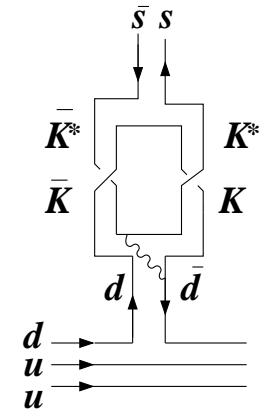

Fig. 4. Non-planar diagram mechanism for the conversion of non-strange to strange quarkonium. 
Table 1

Input (underlined and marked by ${ }^{*}$ ), parameters, post- and predictions of model 1, 2, and 3 (a or b) which follow the input strategy of Refs. [22], [23], and [21], respectively.

\begin{tabular}{|c|c|c|c|c|}
\hline & model 1 & model 2 & model 3a & model $3 \mathrm{~b}$ \\
\hline$m_{\pi}[\mathrm{Mev}]$ & $\underline{138 \pm 2^{*}}$ & $\underline{138 \pm 2^{*}}$ & $\underline{138 \pm 2^{*}}$ & $\underline{138 \pm 2^{*}}$ \\
\hline$m_{K}[\mathrm{MeV}]$ & $\underline{496 \pm 3^{*}}$ & $\underline{496 \pm 3^{*}}$ & $515 \pm 8$ & $550 \pm 12$ \\
\hline$f_{\pi^{0}}[\mathrm{MeV}]$ & $\underline{92 \pm 4^{*}}$ & $\underline{92 \pm 4^{*}}$ & $\underline{92 \pm 4^{*}}$ & $\underline{92 \pm 4^{*}}$ \\
\hline$f_{K^{0}}[\mathrm{MeV}]$ & $127 \pm 5$ & $\underline{113 \pm 5^{*}}$ & $108 \pm 8$ & $101 \pm 6$ \\
\hline$m_{\eta}[\mathrm{MeV}]$ & $\underline{547 \pm 3^{*}}$ & $539 \pm 12$ & $\underline{547 \pm 3^{*}}$ & $\underline{547 \pm 3^{*}}$ \\
\hline$m_{\eta^{\prime}}[\mathrm{MeV}]$ & $\underline{958 \pm 3^{*}}$ & $963 \pm 11$ & $\underline{958 \pm 3^{*}}$ & $\underline{958 \pm 3^{*}}$ \\
\hline$\frac{1}{2} \sqrt{m_{\eta}^{2}+m_{\eta^{\prime}}^{2}}[\mathrm{MeV}]$ & $552 \pm 3$ & $\underline{552 \pm 3^{*}}$ & $552 \pm 3$ & $552 \pm 3$ \\
\hline$m_{a_{0}}[\mathrm{MeV}]$ & $919 \pm 4$ & $1029 \pm 198$ & $1163 \pm 99$ & $1703 \pm 211$ \\
\hline$m_{\kappa}[\mathrm{MeV}]$ & $927 \pm 7$ & $1125 \pm 255$ & $1305 \pm 113$ & $1879 \pm 207$ \\
\hline$m_{\eta_{\mathrm{ns}}}[\mathrm{MeV}]$ & $851 \pm 7$ & $813 \pm 41$ & $778 \pm 17$ & $707 \pm 17$ \\
\hline$m_{\eta_{\mathrm{s}}}[\mathrm{MeV}]$ & $702 \pm 4$ & $746 \pm 41$ & $782 \pm 17$ & $847 \pm 15$ \\
\hline$m_{\eta_{\mathrm{s}-\mathrm{ns}}}^{2}\left[\mathrm{MeV}^{2}\right]$ & $(535 \pm 5)^{2}$ & $(560 \pm 21)^{2}$ & $(556 \pm 5)^{2}$ & $(538 \pm 11)^{2}$ \\
\hline$f_{\eta_{\mathrm{s}}}[\mathrm{MeV}]$ & $161 \pm 7$ & $134 \pm 14$ & $124 \pm 12$ & $109 \pm 8$ \\
\hline$a[\mathrm{MeV}]$ & $65 \pm 3$ & $65 \pm 3$ & $65 \pm 3$ & $65 \pm 3$ \\
\hline$x=\frac{1}{2}\left(\frac{b}{a}-1\right)$ & $0.37 \pm 0.03$ & $0.22 \pm 0.1$ & $0.17 \pm 0.03$ & $0.09 \pm 0.02$ \\
\hline$\lambda$ & $14 \pm 2$ & $47 \pm 52$ & $87 \pm 38$ & $281 \pm 114$ \\
\hline$\beta[\mathrm{MeV}]$ & $-1551 \pm 72$ & $-1698 \pm 104$ & $-1672 \pm 99$ & $-1566 \pm 132$ \\
\hline$\xi\left[\mathrm{MeV}^{2}\right]$ & $(558 \pm 10)^{2}$ & $(375 \pm 170)^{2}$ & $-(246 \pm 36)^{2}$ & $-(969 \pm 133)^{2}$ \\
\hline$\phi_{P}$ & $56.0 \pm 0.5^{\circ}$ & $49.7 \pm 5.7^{\circ}$ & $44.7 \pm 2^{\circ}$ & $34.7 \pm 2^{\circ}$ \\
\hline$\theta_{P}$ & $1.3 \pm 0.5^{\circ}$ & $-5.0 \pm 5.7^{\circ}$ & $-10 \pm 2^{\circ *}$ & $-20 \pm 2^{\circ *}$ \\
\hline$g_{\eta N N}$ & $2.8 \pm 0.5$ & $3.3 \pm 0.6$ & $3.5 \pm 0.7$ & $3.9 \pm 0.7$ \\
\hline$g_{\eta^{\prime} N N}$ & $2.8 \pm 0.4$ & $2.3 \pm 0.6$ & $2.0 \pm 0.7$ & $1.2 \pm 0.7$ \\
\hline$\left(g_{\eta N N}^{2}+g_{\eta^{\prime} N N}^{2}\right) / 4 \pi$ & $1.2 \pm 0.4$ & $1.3 \pm 0.4$ & $1.3 \pm 0.4$ & $1.3 \pm 0.4$ \\
\hline
\end{tabular}

